\title{
Intrinsic Differential Geometry with Geometric Calculus
}

\author{
Hongbo Li and Lina Cao \\ Mathematics Mechanization Key Laboratory \\ Academy of Mathematics and Systems Science \\ Chinese Academy of Sciences \\ Beijing 100080, China
}

\begin{abstract}
Setting up a symbolic algebraic system is the first step in mathematics mechanization of any branch of mathematics. In this paper, we establish a compact symbolic algebraic framework for local geometric computing in intrinsic differential geometry, by choosing only the Lie derivative and the covariant derivative as basic local differential operators. In this framework, not only geometric entities such as the curvature and torsion of an affine connection have elegant representations, but their involved local geometric computing can be simplified.
\end{abstract}

Keywords: Intrinsic differential geometry, Clifford algebra, Mathematics mechanization, Symbolic geometric computing.

\section{Introduction}

Mathematics mechanization focuses on solving mathematical problems with symbolic computation techniques, particularly on mathematical reasoning by algebraic manipulation of mathematical symbols. In differential geometry, the mechanization viz. mechanical theorem proving, is initiated by [7] using local coordinate representation. On the other hand, in modern differential geometry the dominant algebraic framework is moving frames and differential forms [1], which are independent of local coordinates. For mechanical theorem proving in spatial surface theory, [3], [4], [5] proposed to use differential forms and moving frames as basic algebraic tools. It appears that differential geometry benefits from both local coordinates and global invariants [6].

For extrinsic differential geometry, [2] proposed to use Clifford algebra and vector derivative viz. Dirac operator as basic algebraic tools. This framework combines local and global representations, and conforms with the classical framework of vector analysis. The current paper intends to extend this representation to intrinsic differential geometry.

In intrinsic differential geometry, there is already an algebraic system abundant in coordinatefree operators:

Algebraic operators: tensor product $\otimes$, exterior product $\wedge$, interior product $i_{x}$, contraction $\mathcal{C}$, the pairing $\rfloor$ between a vector space and its dual, Riemannian metric tensor $g$, etc. 
Differential operators: exterior differentiation $d$, Lie derivative $\mathcal{L}_{x}$, the bracket $[x, y]$ of tangent vector fields $x, y$, covariant differentiation $D_{x}$, curvature tensor $R$, torsion tensor $T$, etc.

In our opinion, the existing algebraic system contains a lot of redundancy, which may complicate its mechanization. The first task should be to reduce the number of basic operators in the above list, in order to gain both compact representation and effective symbolic manipulation for both local and global geometric computing. This task is fulfilled in this paper.

We propose a compact algebraic framework for local geometric computing in intrinsic differential geometry. We select the following operators as basic ones:

Basic algebraic operators: tensor product $\otimes$, exterior product $\wedge$, interior product ".".

Basic local differential operators: Lie derivative $\mathcal{L}_{a}$, vector derivative $\partial$, covariant differentiations $\delta_{a}$ and $\delta$.

Basic global differential operators: Lie derivative $\mathcal{L}_{x}$, exterior differentiation $d$, covariant differentiation $\delta$.

This framework is suitable for studying intrinsic properties of general vector bundles, affine connections, Riemannian vector bundles, etc, and can be extended to include spin structure and integration. Some typical benefits of this framework include:

1. Exterior differentiation $d$ is equal to the composition of the covariant differentiation $\delta_{a}$ (or $\delta$ in the case of a torsion-free affine connection) and the wedge product.

2. The curvature and torsion tensors can be represented elegantly by covariant differentiation. By these representations, the famous Bianchi identities are given "transparent" algebraic representations.

3. Some geometric theorems can be given simplified proofs, e.g., Schur's Lemma in Riemannian geometry.

This paper is organized as follows. In Section 2 we introduce some terminology and notations. In Section 3 we construct exterior differentiation locally by Lie derivative. In Section 4 we represent curvature tensor, torsion tensor and Bianchi identities locally by covariant differentiation. In Section 5 we represent the curvature tensor of Riemannian manifold explicitly as a 2-tensor of the tangent bivector bundle, and use it to give a simple proof of Schur's Lemma.

\section{Some Terminology and Notations}

We use the same symbol "." to denote the pairing between a vector space and its dual space, the interior product of a tangent vector field and a differential form, and the inner product induced by a pseudo-Riemannian metric tensor. The explanation is that if no metric tensor occurs, then the dot symbol denotes the interior product which includes the pairing naturally; if there is a fixed pseudo-Riemannian metric tensor, then the tangent space and 
cotangent space are identified, so are the pairing and the inner product induced by the metric. The latter identification is further justified by the fact that in Riemannian geometry, we use only the Levi-Civita covariant differentiation, which preserves both the inner product and the pairing.

In this paper, we use the following extension [2] of the pairing "." from $\mathcal{V}$ and $\mathcal{V}^{*}$ to their Grassmann spaces $\Lambda(\mathcal{V})$ and $\Lambda\left(\mathcal{V}^{*}\right)$ : for vectors $x_{i} \in \mathcal{V}$ and $y^{j} \in \mathcal{V}^{*}$,

$$
\left(x_{1} \wedge \cdots \wedge x_{r}\right) \cdot\left(y^{1} \wedge \cdots \wedge y^{s}\right)= \begin{cases}\left(x _ { 1 } \cdot \left(x _ { 2 } \cdot \left(\cdots\left(x_{r} \cdot\left(y^{1} \wedge \cdots \wedge y^{s}\right) \cdots\right),\right.\right.\right. & \text { if } r \leq s \\ \left.\left.\left.\left(\cdots\left(x_{1} \wedge \cdots \wedge x_{r}\right) \cdot y^{1}\right) \cdot y^{2}\right) \cdots\right) \cdot y^{s}\right), & \text { if } r>s\end{cases}
$$

The topic "vector derivative" is explored in detail in [2]. Let $\mathcal{V}$ and $\mathcal{V}^{*}$ be an $n \mathrm{D}$ vector space and its dual space, Let $\left\{a_{i} \mid i=1 \ldots n\right\}$ and $\left\{a_{j}\right\}$ be a pair of dual bases of $\mathcal{V}$ and $\mathcal{V}^{*}$ respectively. The vector derivative at a point $x \in \mathcal{V}$ is the following $\mathcal{V}$-valued first-order differential operator:

$$
\partial_{x}=\left.\sum_{i=1}^{n} a^{i} \frac{\partial}{\partial a_{i}}\right|_{x} .
$$

Here $\partial /\left.\partial a_{i}\right|_{x}$ denotes the directional derivative at point $x$. Obviously, $\partial=\partial_{x}$ is independent of the basis $\left\{a_{i}\right\}$ chosen, and for any vector $a$,

$$
a \cdot \partial=\partial / \partial a, \quad a=\sum_{i=1}^{n}\left(a \cdot a^{i}\right) a_{i} .
$$

Henceforth we consider an $n \mathrm{D}$ differentiable manifold $M$. The tangent and cotangent bundles of $M$ are denoted by $T M$ and $T^{*} M$ respectively. The set of smooth tangent and cotangent vector fields on $M$ are denoted by $\Gamma(T M)$ and $\Gamma\left(T^{*} M\right)$ respectively.

The Lie derivatives of smooth scalar field $f$, tangent vector field $y$, and cotangent vector field $\omega$ with respect to a smooth tangent vector field $x$, are defined by

$$
\begin{array}{ll}
\mathcal{L}_{x} f & =x(f), \\
\mathcal{L}_{x} y & =[x, y] \\
y \cdot \mathcal{L}_{x}(\omega) & =\mathcal{L}_{x}(y \cdot \omega)-\omega \cdot \mathcal{L}_{x} y .
\end{array}
$$

Here $[x, y]$ is the bracket defined by

$$
[x, y](f)=x(y(f))-y(x(f)), \forall f \in C^{\infty}(M) .
$$

Below we introduce two notations. Let $\pi$ be a differential operator taking values in $\Lambda\left(\Gamma\left(T^{*} M\right)\right)$. Then in $\pi \wedge u \wedge v$, where $u, v \in \Lambda\left(\Gamma\left(T^{*} M\right)\right)$, the differentiation is carried out to the right hand side of $\pi$, i.e., to both $u$ and $v$. If we want the differentiation be made only to $u$, we use $\pi \wedge \dot{u} \wedge v$ to denote this, else if we want it be made only to $v$, we use $\pi \wedge \check{u} \wedge v$ to denote this.

If $u$ itself is a differential operator taking values in $\Lambda\left(\Gamma\left(T^{*} M\right)\right)$, then it has two parts: the $\Lambda\left(\Gamma\left(T^{*} M\right)\right)$-part and the scalar-valued differential operator part. If the differentiation in $\pi$ is made only to the $\Lambda\left(\Gamma\left(T^{*} M\right)\right)$-part of $u$, we use $\pi \wedge \dot{u} \wedge v$ to denote this. If the differentiation is not made to the $\Lambda\left(\Gamma\left(T^{*} M\right)\right)$-part of $u$, we use $\pi \wedge \check{u} \wedge v$ to denote this. The scalar-valued 
differentiation parts $s(\pi), s(u)$ of $\pi, u$ always act on $v$ in the manner $s(\pi) s(u) v$. If $v$ is also a $\Lambda\left(\Gamma\left(T^{*} M\right)\right)$-valued differential operator, and the differentiation in $\pi$ is made only to the $\Lambda\left(\Gamma\left(T^{*} M\right)\right)$-part of $v$, we use $\pi \wedge u \wedge \dot{v}$ or $\pi \wedge \check{u} \wedge \dot{v}$ to denote this, depending on how $u$ is differentiated.

\section{Exterior Differentiation}

Definition 3.1 A local natural basis $\left\{a_{i}\right\}$ of $\Gamma(T M)$ at $x \in M$ is a set of $n$ smooth tangent vector fields defined in a neighborhood $N$ of $x$, such that there exists a local coordinate system $\left\{u_{i}\right\}$ of $N$ with the property

$$
a_{i}(f)=\frac{\partial f}{\partial u_{i}}, \forall f \in C^{\infty}(N) .
$$

Let $\left\{a_{i}\right\}$ be a local basis of $\Gamma(T M)$, and let $\left\{a^{i}\right\}$ be its dual basis in $\Gamma\left(T^{*} M\right)$. Define

$$
d^{a}=\sum_{i=1}^{n} a^{i} \wedge \mathcal{L}_{a_{i}}=\sum_{i=1}^{n} a^{i} \mathcal{L}_{a_{i}} \wedge .
$$

Lemma 3.2 (1) Let $\left\{a_{i}\right\}$ be a local basis of $\Gamma(T M)$. Then it is natural if only if $\left[a_{i}, a_{j}\right]=0$ for any $1 \leq i<j \leq n$.

(2) $\left(d^{a}\right)^{2}=0$ if and only if $\left\{a_{i}\right\}$ is natural.

Proof. We only prove the second part. By the definition of $d^{a}$, to prove $\left(d^{a}\right)^{2}=0$ we only need to prove $\left(d^{a}\right)^{2} f=0$ for any $f \in C^{\infty}(M)$.

$$
\begin{aligned}
\left(d^{a}\right)^{2} f & =\sum_{i, j=1}^{n} a^{i} \wedge \mathcal{L}_{a_{i}}\left(a^{j} a_{j}(f)\right) \\
& =\sum_{i, j=1}^{n}\left(a^{i} \wedge a^{j} a_{i}\left(a_{j}(f)\right)\right)+a_{j}(f) a^{i} \wedge \mathcal{L}_{a_{i}}\left(a^{j}\right) \\
& =\sum_{i<j}\left[a_{i}, a_{j}\right](f) a^{i} \wedge a^{j}-\sum_{i, j, k=1}^{n} a_{j}(f) a^{i} \wedge\left(\left[a_{i}, a_{k}\right] \cdot a^{j}\right) a^{k} \\
& =\sum_{i<j}\left[a_{i}, a_{j}\right](f) a^{i} \wedge a^{j}-\sum_{i, k=1}^{n}\left[a_{i}, a_{k}\right](f) a^{i} \wedge a^{k} \\
& =-\sum_{i<j}\left[a_{i}, a_{j}\right](f) a^{i} \wedge a^{j} .
\end{aligned}
$$

Thus $\left(d^{a}\right)^{2} f=0$ if and only if $\left[a_{i}, a_{j}\right](f)=0$, i.e., $\left\{a_{i}\right\}$ is natural.

Proposition $3.3 d^{a}$ is independent of the local natural basis $\left\{a_{i}\right\}$. It is defined globally on $M$, called the exterior differentiation operator of $M$, denoted by $d$.

Proof. It is easy to prove that $\left.d^{a}\right|_{C^{\infty}(M)}$ is independent of $a_{i}$. So we only need to prove that $\left.d^{a}\right|_{\Gamma\left(T^{*} M\right)}$ is independent of $a_{i}$. The latter is true because

$$
d^{a}\left(f d^{a}(g)\right)=d^{a} f \wedge d^{a} g+f\left(d^{a}\right)^{2} g=d^{a} f \wedge d^{a} g
$$


is independent of $\left\{a_{i}\right\}$, for any $f, g \in C^{\infty}(M)$.

\section{I}

For local calculus, we can further decompose $d$ into two parts: the wedge product " $\wedge$ ", and the $\Gamma\left(T^{*} M\right)$-valued local differential operator

$$
\delta_{a}=\sum_{i=1}^{n} a^{i} \mathcal{L}_{a_{i}} .
$$

This operator depends on the local basis $\left\{a_{i}\right\}$. The benefit of introducing $\delta_{a}$ can be seen from the following local expression of $[x, y]$.

Proposition 3.4 (1) For any local natural basis $\left\{a_{i}\right\}$,

$$
\delta_{a} \wedge \delta_{a}=0 .
$$

(2) For any smooth local tangent vector fields $x, y$,

$$
[x, y]=x \cdot \delta_{a} y-y \cdot \delta_{a} x .
$$

Proof. We only prove the second part. For any $f \in C^{\infty}(M)$,

$$
\begin{aligned}
{[x, y](f) } & =x \cdot \delta_{a}\left(y \cdot \delta_{a} f\right)-y \cdot \delta_{a}\left(x \cdot \delta_{a} f\right) \\
& =\left(x \cdot \delta_{a} y\right) \cdot \delta_{a} f+y \cdot\left(x \cdot \delta_{a} \delta_{a} f\right)-\left(y \cdot \delta_{a} x\right) \cdot \delta_{a} f-x \cdot\left(y \cdot \delta_{a} \delta_{a} f\right) \\
& =\left(x \cdot \delta_{a} y-y \cdot \delta_{a} x\right)(f)-(x \wedge y) \cdot\left(\delta_{a} \wedge \delta_{a}\right) f \\
& =\left(x \cdot \delta_{a} y-y \cdot \delta_{a} x\right)(f) .
\end{aligned}
$$

\section{Connection on a Vector Bundle}

A connection of a vector bundle $E$ over $M$ is denoted by $D$. Its covariant derivative with respect to a vector field $a$ is denoted by $D_{a}$. As in the case of exterior differentiation, we can decompose $D$ into two parts: the tensor product " $\otimes$ ", and the $\Gamma\left(T^{*} M\right)$-valued local differential operator

$$
\delta=\sum_{i=1}^{n} a^{i} D_{a_{i}}
$$

where $\left\{a_{i}\right\}$ is a local basis of $\Gamma(T M)$. The difference is that here the operator $\delta$ is independent of the basis $\left\{a_{i}\right\}$ chosen, called the covariant differentiation operator. We have

$$
D=\delta \otimes, \quad D_{a}=a \cdot \delta .
$$

The first benefit of introducing $\delta$ is that we can add it up with any differential form $\omega$. The sum is a new connection, and the new covariant derivative is $a \cdot(\delta+\omega)$. The second benefit is its intrinsic characterization of the curvature tensor, and in the case of affine connection, also the torsion tensor.

The scalar-valued operator $(x \wedge y) \cdot(\delta \wedge \delta)$ represents the difference between the two compositions $D_{x} D_{\breve{y}}$ and $D_{y} D_{\breve{x}}$ of the differentiations $D_{x}$ and $D_{y}$. So $\delta \wedge \delta$ measures the asymmetry of two successive covariant derivatives. In this section, we decompose this asymmetry into two parts: the curvature part and the torsion part. This characterization clarifies the intrinsic relationship between an affine connection and its curvature and torsion tensors. 
Definition 4.1 The differential operator

$$
R(x, y)=D_{x} D_{y}-D_{y} D_{x}-D_{[x, y]}, \quad \forall x, y \in \Gamma(T M)
$$

is called the curvature operator of the connection. The following $(3,1)$-tensor is called the curvature tensor of the connection:

$$
R(x, y, z, \omega)=\omega \cdot\left(\left(D_{x} D_{y}-D_{y} D_{x}-D_{[x, y]}\right) z\right), \quad \forall x, y, z \in \Gamma(T M), \omega \in \Gamma\left(T^{*} M\right) .
$$

We use the same symbol $R$ with different number of arguments to denote both the curvature operator and the curvature tensor.

Proposition 4.2 The curvature operator has the following local representation with respect to a local natural basis $\left\{a_{i}\right\}$ :

$$
R(x, y)=-(x \wedge y) \cdot(\delta \wedge \check{\delta}) .
$$

Proof. By $R\left(a_{i}, a_{j}\right)=a_{i} \cdot \delta\left(a_{j} \cdot \delta\right)-a_{j} \cdot \delta\left(a_{i} \cdot \delta\right)$ and

$$
\delta \wedge \check{\delta}=-\sum_{i=1}^{n} a^{i} \wedge \delta\left(a_{i} \cdot \delta\right)=\sum_{i, j=1}^{n}\left(a^{i} \wedge a^{j}\right) a_{i} \cdot \delta\left(a_{j} \cdot \delta\right),
$$

we get $\delta \wedge \check{\delta}=\sum_{i<j}\left(a^{i} \wedge a^{j}\right) R\left(a_{i}, a_{j}\right)$. From this and the fact that $R(x, y)$ is a function of $x \wedge y$, we obtain (4.15).

Corollary 4.3 $\delta \wedge \check{\delta}$ is independent of $\left\{a_{i}\right\}$ as long as the local basis is natural. Furthermore, $\left.\delta \wedge \check{\delta}\right|_{C^{\infty}(M)}=0$.

Definition 4.4 The torsion tensor of an affine connection $D$ is defined by

$$
T(x, y)=D_{x} y-D_{y} x-[x, y], \quad \forall x, y \in \Gamma(T M) .
$$

The dual connection and dual covariant differentiation of $D$ and $\delta$ are defined by their actions on a local natural basis $\left\{a_{i}\right\}$ :

$$
\bar{D}_{a_{i}}\left(a_{j}\right)=D_{a_{j}} a_{i}, \quad a_{i} \cdot \bar{\delta} a_{j}=a_{j} \cdot \delta a_{i} .
$$

In fact, for any tangent vector fields $x, y$,

$$
\begin{aligned}
& \bar{D}_{x}(y)=y \cdot \delta x+[x, y] \\
& T(x, y)=x \cdot(\delta-\bar{\delta} y) .
\end{aligned}
$$

Proposition 4.5 For any tangent vector fields $x, y$ and cotangent vector field $\omega$,

$$
\begin{aligned}
& T(x, y) \cdot \delta=(x \wedge y) \cdot(\delta \wedge \dot{\delta}) \\
& T(x, y) \cdot \omega=(x \wedge y) \cdot(\delta \wedge \omega-d \omega) .
\end{aligned}
$$

In particular, $\delta \wedge \dot{\delta}$ is independent of $\left\{a_{i}\right\}$ as long as the local basis is natural.

Corollary 4.6 1. $d=\delta \wedge$ if and only if $\delta$ is torsion-free. 
2. If $\delta$ is torsion-free, then

$$
\overbrace{\delta \wedge \cdots \wedge \delta}^{r}=\sum_{i_{1}, \cdots, i_{r}=1}^{n} a^{i_{1}} \wedge \cdots \wedge a^{i_{r}} a_{i_{1}} \cdot \delta \cdots a_{i_{r}} \cdot \delta .
$$

Proposition 4.7 For a local natural basis $\left\{a_{i}\right\}$, the curvature and torsion of the connection $\delta_{a}=\sum_{i=1}^{n} a^{i} \mathcal{L}_{a_{i}}$ are both zero. It is called a local natural affine connection.

Theorem 4.8 [Commutation Formula]

$$
(x \wedge y) \cdot(\delta \wedge \delta)=T(x, y) \cdot \delta-R(x, y) .
$$

Proof. In a local natural basis $\left\{a_{i}\right\}$, the conclusion follows (4.15), (4.20) and the Lebniz rule $\delta \wedge \delta=\delta \wedge \dot{\delta}+\delta \wedge \check{\delta}$. If $\left\{a_{i}\right\}$ is not natural, then

$$
-R=\delta \wedge \check{\delta}+d \delta, \quad T \cdot \delta=\delta \wedge \dot{\delta}-d \delta .
$$

The relation (4.22) still holds.

\section{I}

When $T=0$, then $R=\delta \wedge \delta$. This representation crystalizes the famous Bianchi identities as follows.

Proposition 4.9 [Bianchi] Let $\delta$ be torsion-free, $R$ be its curvature. Let $\left\{a_{i}\right\}$ be a local natural basis of $\Gamma(T M)$. The components of $R$ and $D R$ with respect to $\left\{a_{i}\right\}$ and its dual basis $\left\{a^{i}\right\}$ are denoted by $R_{i k l}^{j}, R_{i k l, h}^{j}$ respectively. Then

$$
\begin{aligned}
& R_{j k l}^{i}+R_{k l j}^{i}+R_{l j k}^{i}=0 \\
& R_{j k l, h}^{i}+R_{j l h, k}^{i}+R_{j h k, l}^{i}=0
\end{aligned}
$$

Proof. We have $-R_{j k l}^{i}=a^{i} \cdot\left(\left(a_{k} \wedge a_{l}\right) \cdot(\delta \wedge \check{\delta}) a_{j}\right)$, and the relation

$$
\left(a_{k} \wedge a_{l}\right) \cdot(\delta \wedge \check{\delta})\left(a^{i} \cdot a_{j}\right)=a^{i} \cdot\left(\left(a_{k} \wedge a_{l}\right) \cdot(\delta \wedge \check{\delta}) a_{j}\right)+a_{j} \cdot\left(\left(a_{k} \wedge a_{l}\right) \cdot(\delta \wedge \check{\delta}) a^{i}\right)=0 .
$$

Then

$$
\begin{aligned}
R_{j k l}^{i}+R_{k l j}^{i}+R_{l j k}^{i}= & a_{j} \cdot\left(\left(a_{k} \wedge a_{l}\right) \cdot(\delta \wedge \check{\delta}) a^{i}\right)+a_{k} \cdot\left(\left(a_{l} \wedge a_{j}\right) \cdot(\delta \wedge \check{\delta}) a^{i}\right) \\
& +a_{l} \cdot\left(\left(a_{j} \wedge a_{k}\right) \cdot(\delta \wedge \check{\delta}) a^{i}\right) \\
= & \left(a_{j} \wedge a_{k} \wedge a_{l}\right) \cdot\left(\delta \wedge \check{\delta} \wedge a^{i}\right) .
\end{aligned}
$$

The first Bianchi identity is equivalent to

$$
\delta \wedge \check{\delta} \wedge a^{i}=0
$$

when $T=0$ and $\left\{a_{i}\right\}$ is natural. It follows $d^{2}=0$.

From

$$
R_{j k l, h}^{i}=\left(a_{h} \cdot \delta R\right)\left(a_{k}, a_{l}, a_{j}, a^{i}\right)=\left(a_{k} \wedge a_{l}\right) \cdot\left(a_{h} \cdot \delta R\right)\left(a_{j}, a^{i}\right),
$$

we get

$$
R_{j k l, h}^{i}+R_{j l h, k}^{i}+R_{j h k, l}^{i}=\left(a_{k} \wedge a_{l} \wedge a_{h}\right) \cdot(\delta \wedge \dot{R})\left(a_{j}, a^{i}\right) .
$$

The second Bianchi identity is equivalent to

$$
\delta \wedge \dot{R}=\delta \wedge(\delta \wedge \check{\delta})^{\cdot}=0
$$

When $T=0$ and $\left\{a_{i}\right\}$ is natural. It follows $\delta \wedge \dot{\delta}=0$ and

$$
\delta \wedge(\delta \wedge \check{\delta})^{\cdot}=\delta \wedge \dot{\delta} \wedge(\check{\delta})^{\vee}+\delta \wedge \check{\delta} \wedge(\check{\delta})^{.}
$$




\section{Riemannian geometry}

The Levi-Civita connection $\delta$ of a generalized Riemannian manifold is the unique torsionfree connection preserving the metric tensor. Its curvature tensor $R$ induces a new differential operator, denoted by $R^{\prime}$ :

$$
R^{\prime}(z, \omega)(x, y)=R(x, y, z, \omega) .
$$

Since $R^{\prime}(z, \omega)$ is a $C^{\infty}(M)$-linear function on $\Lambda^{2}(\Gamma(T M))$, there exists a unique $\Omega(z, \omega) \in$ $\Lambda^{2}\left(\Gamma\left(T^{*}(M)\right)\right)$ such that $R^{\prime}(z, \omega)(x, y)=(x \wedge y) \cdot \Omega(z, \omega)$. Below we compute $\Omega$.

By (4.15),

$$
\Omega(z, \omega)=-\delta \wedge \check{\delta}(\dot{z} \cdot \omega)=\delta \wedge \check{\delta}(\dot{\omega} \cdot z)=z \cdot(\delta \wedge \check{\delta} \wedge \omega)-(z \cdot(\delta \wedge \check{\delta})) \wedge \omega,
$$

so for any local natural basis $\left\{a_{i}\right\}$,

$$
\begin{aligned}
\Omega\left(a_{i}, a_{j}\right) & =-\left(a_{i} \cdot(\delta \wedge \check{\delta})\right) \wedge a_{j} \\
& =-\sum_{k=1}^{n} a^{k} \wedge\left(a_{i} \cdot \delta a_{k} \cdot \delta a_{j}-a_{k} \cdot \delta a_{i} \cdot \delta a_{j}\right) \\
& =-\sum_{k=1}^{n} a^{k} \wedge\left(a_{i} \cdot \delta a_{j} \cdot \delta a_{k}\right)+\delta \wedge\left(a_{i} \cdot \delta a_{j}\right) .
\end{aligned}
$$

Since $\Omega\left(a_{i}, a_{j}\right)=-\Omega\left(a_{j}, a_{i}\right)$ and $a_{i} \cdot \delta a_{j}=a_{j} \cdot \delta a_{i}$,

$$
\Omega\left(a_{i}, a_{j}\right)=-\frac{1}{2} \sum_{k=1}^{n} a^{k} \wedge R\left(a_{i}, a_{j}\right) a_{k} .
$$

A direct corollary of $(5.28)$ is that $R=R^{\prime}$, i.e.,

$$
R\left(a_{k}, a_{l}\right)\left(a_{i}, a_{j}\right)=R^{\prime}\left(a_{i}, a_{j}\right)\left(a_{k}, a_{l}\right)=\left(a_{k} \wedge a_{l}\right) \cdot \Omega\left(a_{i}, a_{j}\right)=R\left(a_{i}, a_{j}\right)\left(a_{k}, a_{l}\right) .
$$

Because of this, we can identify the curvature tensor with the following symmetric 2-tensor in the space $\Lambda^{2}(\Gamma(T M))$, still denoted by $R$ :

$$
R(x \wedge y, z \wedge \omega)=R(x, y)(z, \omega)=R(x, y, z, \omega) .
$$

Proposition 5.1 For any local basis $\left\{a_{i}\right\}$ which is not necessarily natural,

$$
R=-\frac{1}{2} \sum_{k=1}^{n}\left(a^{k} \wedge \delta\right) \otimes\left(\check{\delta} \wedge a_{k}\right)=-\frac{1}{2}(\delta \wedge \check{\delta}) \otimes\left(\sum_{k=1}^{n} \dot{a}_{k} \wedge a^{k}\right)=-\frac{1}{2}(\delta \wedge \check{\delta}) \otimes\left(\sum_{k=1}^{n} \dot{a}^{k} \wedge a_{k}\right) .
$$

Let $B_{2}$ be a 2-blade of $T_{p} M, p \in M$. Then

$$
K\left(B_{2}\right)=R\left(B_{2}^{-1}, B_{2}\right)
$$

is called the sectional curvature at point $p$.

Proposition 5.2 At every point $p$ of $M, R$ is determined by $K$. 
Proof. Let $\partial$ be the vector derivative in the vector space $\Lambda^{2}\left(T_{p} M\right)$. For any $A_{2} \in$ $\Lambda^{2}(\Gamma(T M))$, by the multilinearity of $R$, we have

$$
A_{2} \cdot \partial\left(R\left(B_{2}^{-1}, B_{2}\right)\right)=2 \frac{R\left(A_{2}, B_{2}\right) B_{2} \cdot B_{2}-R\left(B_{2}, B_{2}\right) A_{2} \cdot B_{2}}{\left(B_{2} \cdot B_{2}\right)^{2}},
$$

so

$$
R\left(A_{2}, B_{2}\right)=K A_{2} \cdot B_{2}+\frac{1}{2}\left(B_{2} \cdot B_{2}\right)\left(A_{2} \cdot \partial K\right)
$$

\section{I}

If at $p \in M, K\left(B_{2}\right)$ is independent of $B_{2}$, we say $M$ is isotropic at $p$. If $M$ is isotropic at every point and $K=K_{p}$ is independent of $p$, we call $M$ a constant-curvature space.

Proposition 5.3 [F. Schur] If $M$ is an $n$ D connected Riemannian manifold $(n>2)$ and is isotropic at every point, then it is a constant-curvature space.

Proof. We only need to prove that $K=K_{p}$ is independent of $p$. First, since $K_{p}$ is constant at $\Lambda^{2}\left(T_{p} M\right)$, i.e., $A_{2} \cdot \partial K_{p}=0$ for any $A_{2} \in \Lambda\left(\Gamma\left(T_{p} M\right)\right)$, by (5.32), we have $R\left(A_{2}, B_{2}\right)=K_{p} A_{2} \cdot B_{2}$ for any $B_{2} \in \Lambda\left(\Gamma\left(T_{p} M\right)\right)$, so

$$
R\left(B_{2}\right)=K B_{2} .
$$

Below we compute $\delta \wedge R\left(B_{2}\right)$ using the relation $\delta \wedge \dot{R}=0$ :

$$
\delta \wedge R\left(B_{2}\right)=\delta \wedge \dot{R}\left(B_{2}\right)+\delta \wedge \check{R}\left(\dot{B}_{2}\right)=K \delta \wedge B_{2} .
$$

On the other hand, by differentiating the right-hand side of (5.33) directly, we get

$$
\delta \wedge R\left(B_{2}\right)=\delta \wedge\left(K B_{2}\right)=(\delta \dot{K}) \wedge B_{2}+K \delta \wedge B_{2} .
$$

So for any $B_{2} \in \Lambda^{2}\left(\Gamma\left(T_{p} M\right)\right),(\delta \dot{K}) \wedge B_{2}=0$. Since the dimension of $\Lambda^{2}\left(\Gamma\left(T_{p} M\right)\right)$ is greater than one, it must be that $\delta K=0$ for any $p$, i.e., $K$ is constant over $M$.

\section{Conclusion}

In this paper, we set up a compact symbolic algebraic system for local geometric computing in intrinsic differential geometry. In this system, the exterior differentiation, the curvature tensor of a connection, the torsion tensor of an affine connection, the Bianchi identities of a torsion-free affine connection, and the curvature tensor of a Levi-Civita connection are given elegant representations, which can be used to simplify the involved geometric computing, e.g. in the proof of Schur's Lemma. The idea behind the system is the use of vector derivative and Clifford algebra. Our investigation shows that this is a promising research direction for both mathematics mechanization and differential geometry.

\section{References}

[1] S. S. Chern, W.-H. Chen and K.-S. Lan (1999): Lectures on Differential Geometry, World Scientific, Singapore.

[2] D. Hestenes and G. Sobczyk (1984): Clifford Algebra to Geometric Calculus, D. Reidel, Dordrecht, Boston. 
[3] H. Li (1995). Automated reasoning with differential forms. In: Proc. ASCM'95, Scientists Inc., Tokyo, pp. 29-32.

[4] H. Li (1997). On mechanical theorem proving in differential geometry - local theory of surfaces. Science in China A, 40(4): 350-356.

[5] H. Li (2000). Mechanical theorem proving in differential geometry. In: Mathematics Mechanization and Applications, X.-S. Gao and D. Wang (eds.), Academic Press, London, pp. 147-174.

[6] T. J. Willmore (1993): Riemannian Geometry, Clarendon Press, Oxford, New York.

[7] W.-T. Wu (1979): On the mechanization of theorem-proving in elementary differential geometry. Scientia Sinica (Math Suppl. I): 94-102. 